\title{
A Theorem on Improving Regularity of Minimizing Sequences by Reverse Hölder Inequalities
}

\author{
BAISHENG YAN \& ZHENGFANG ZHOU
}

\section{Introduction}

The use of reverse Hölder inequalities pioneered by Gehring's celebrated lemma [5] in the theory of quasiconformal mappings has been well adapted in the calculus of variations for obtaining regularity of minimizers of integral functionals with certain natural growth conditions [6]. In this paper we elaborate upon some ideas of our recent paper [16] to prove a theorem on improving regularity of minimizing sequences of a family of integral functionals that do not satisfy the usual growth conditions but satisfy instead a uniform integral coercivity condition as given by (1.4) below. As an important application, we also prove a stability result on the strong convergence of the so-called weakly almost conformal mappings in $W^{1, p}\left(\Omega ; \mathbf{R}^{n}\right)$ for certain $p$ below the dimension $n$. See also $[4 ; 7 ; 11 ; 13 ; 14 ; 16]$.

We begin with some notation. Let $\mathcal{M}^{n \times m}$ be the space of all real $n \times m$-matrices with norm $|X|$ defined by $|X|^{2}=\operatorname{tr}\left(X^{T} X\right)$. For $p \geq 1$ and a domain $D$ in $\mathbf{R}^{m}$, let $W^{1, p}\left(D ; \mathbf{R}^{n}\right)$ be the usual Sobolev space of $L^{p}$-integrable maps $u: D \rightarrow \mathbf{R}^{n}$ having $L^{p}$-integrable gradients $(\nabla u)_{i j}=\partial u^{i} / \partial x_{j}$ for $1 \leq i \leq n$ and $1 \leq j \leq m$.

Let $\mathcal{K}$ be a closed subset of $\mathcal{M}^{n \times m}$, and let $d_{\mathcal{K}}(X)=\inf _{A \in \mathcal{K}}|X-A|$ be the distance function to $\mathcal{K}$. In this paper, we shall always assume that $d_{\mathcal{K}}$ satisfies the following condition:

$$
d_{\mathcal{K}}(\lambda X) \leq K_{0}\left(d_{\mathcal{K}}(X)+1\right), \quad X \in \mathcal{M}^{n \times m}, 0 \leq \lambda \leq 1 .
$$

Note that condition (1.1) is satisfied if $\mathcal{K}$ is a cone or a bounded set.

We consider the integral functionals $I_{p}(u ; D)$ defined by

$$
I_{p}(u ; D)=\int_{D} d_{\mathcal{K}}^{p}(\nabla u(x)) d x .
$$

The natural admissible space for $I_{p}(u ; D)$ is $W^{1, p}\left(D ; \mathbf{R}^{n}\right)$, but we shall often consider $I_{p}(u ; D)$ for all $u \in W_{\text {loc }}^{1,1}\left(D ; \mathbf{R}^{n}\right)$.

Throughout this paper, we assume that $1 \leq \alpha \leq \beta<\infty$ are given numbers, that $\Omega \subset \subset D_{0}$ are bounded smooth domains in $\mathbf{R}^{m}$, and that $u_{0}$ is a given map in $W_{\text {loc }}^{1, \alpha}\left(D_{0} ; \mathbf{R}^{n}\right)$ satisfying 


$$
\nabla u_{0}(x) \in \mathcal{K} \text { a.e. } x \in D_{0} .
$$

We shall also assume that $\mathcal{K}$ satisfies the following condition.

Uniform Integral Coercivity. There exist constants $\Gamma_{0}>0$ and $\Gamma_{1} \geq 0$ depending on $n, \alpha, \beta$, and $\mathcal{K}$ such that, for every ball $B \subset \mathbf{R}^{m}$,

$$
\int_{B} d_{\mathcal{K}}^{p}(\nabla \phi) \geq \Gamma_{0} \int_{B}\left(|\nabla \phi|^{p}-\Gamma_{1}\right) \quad \forall p \in[\alpha, \beta], \quad \phi \in C_{0}^{\infty}\left(B ; \mathbf{R}^{n}\right) .
$$

We remark that condition (1.4) is satisfied for all compact sets $\mathcal{K}$. Note also that from (1.4) one easily sees that, for any bounded domain $D$ and $\phi \in C_{0}^{\infty}\left(D ; \mathbf{R}^{n}\right)$,

$$
\int_{D} d_{\mathcal{K}}^{p}(\nabla \phi) \geq \Gamma_{0} \int_{D}|\nabla \phi|^{p}-\left(\Gamma_{0} \Gamma_{1}+d_{\mathcal{K}}^{p}(0)\right)|B|
$$

for all $p \in[\alpha, \beta]$ and balls $B$ containing $D$.

The main result of this paper is the following theorem.

THEOREM 1.1. There exists a constant $\bar{\varepsilon}>0$ depending only on $n, \alpha, \beta$, and $\mathcal{K}$ such that, for any sequence $\left\{u_{j}\right\}$ in $W^{1, \alpha}\left(\Omega ; \mathbf{R}^{n}\right)$ that converges weakly to $u_{0}$ and satisfies $\lim _{j \rightarrow \infty} I_{\alpha}\left(u_{j} ; \Omega\right)=0$, there exist a sequence $\left\{v_{k}\right\}$ in $W_{\mathrm{loc}}^{1, \beta+\bar{\varepsilon}}\left(D_{0} ; \mathbf{R}^{n}\right)$ and a subsequence $\left\{u_{j_{k}}\right\}$ such that $v_{k}=u_{0}$ in $D_{0} \backslash \Omega, v_{k} \rightarrow u_{0}$ in $W_{\mathrm{loc}}^{1, \beta+\bar{\varepsilon}}\left(D_{0} ; \mathbf{R}^{n}\right)$, and

$$
\lim _{k \rightarrow \infty}\left(I_{\beta+\bar{\varepsilon}}\left(v_{k} ; D_{0}\right)+\int_{\Omega}\left|\nabla v_{k}-\nabla u_{j_{k}}\right|\right)=0 .
$$

Remarks. (1) The new sequence $\left\{v_{k}\right\}$ is not only a minimizing sequence of the functional $I_{\alpha}(u ; \Omega)$ but also a minimizing sequence for all functionals $I_{p}(u ; \Omega)$ with $p \in[\alpha, \beta+\bar{\varepsilon}]$; moreover, it has a higher integrability than $\left\{u_{j}\right\}$.

(2) From this theorem we obtain that the uniform $L^{p}$-coercivity (1.4) implies a higher regularity for solutions of first-order system (1.3) in $W_{\text {loc }}^{1, \alpha}\left(D_{0} ; \mathbf{R}^{n}\right)$, that is, $u_{0} \in W_{\text {loc }}^{1, \beta+\bar{\varepsilon}}\left(D_{0} ; \mathbf{R}^{n}\right)$. Also, by the Sobolev embedding, if $\beta \geq n$ then the sequence $\left\{v_{k}\right\}$ can be chosen in the Hölder space $C_{\text {loc }}^{0, \mu}\left(D_{0} ; \mathbf{R}^{n}\right)$ for some $\mu \in$ $(0,1)$.

(3) As an important application, we shall prove a new strong convergence theorem (Theorem 5.1) for the weakly almost conformal mappings in $W^{1, p}\left(\Omega ; \mathbf{R}^{n}\right)$ with certain $p<n$. See also [11].

The paper is organized as follows. In Section 2 we prove a preliminary lemma that will be used in later sections. In Section 3, we use a version of Ekeland's variational principle and Caccioppoli-type estimates to obtain the reverse Hölder inequality with increasing supports; then a higher regularity follows from the well-known Gehring's lemma. We then prove the main result, Theorem 1.1, in Section 4. Finally, we give an application in Section 5 by proving a strong convergence for the weakly almost conformal mappings.

ACKNOWLEDGMENTs. The authors would like to acknowledge stimulating discussions with Michael Frazier regarding the reverse Hölder inequality. We also thank the referee for many useful suggestions. 


\section{A Useful Lemma}

Before we proceed with the proof of Theorem 1.1, we prove the following useful lemma.

LEMma 2.1. Let $p \geq 1$, and let $\left\{u_{j}\right\}$ be a sequence in $W^{1, p}\left(\Omega ; \mathbf{R}^{n}\right)$ that converges weakly to $u$ and satisfies $\lim _{j \rightarrow \infty} I_{p}\left(u_{j} ; \Omega\right)=0$. Suppose the weak limit $u$ extends to a map in $W^{1, p}\left(D ; \mathbf{R}^{n}\right)$ for some $D$ with $\Omega \subset \subset D$ that satisfies $\nabla u(x) \in$ $\mathcal{K}$ for a.e. $x \in D$. Then, there exist a sequence $\left\{w_{k}\right\}$ in $W^{1, p}\left(D ; \mathbf{R}^{n}\right)$ and a subsequence $\left\{u_{j_{k}}\right\}$ such that $w_{k}=u$ in $D \backslash \Omega, w_{k} \rightarrow u$ weakly in $W^{1, p}\left(D ; \mathbf{R}^{n}\right)$, and

$$
I_{p}\left(w_{k} ; D\right)+\int_{\Omega}\left|\nabla u_{j_{k}}-\nabla w_{k}\right| \leq \frac{k^{-2}}{2} \quad \forall k=1,2, \ldots
$$

Proof. The proof is standard. Let $\Omega_{k}=\{x \in \Omega \mid \operatorname{dist}(x, \partial \Omega)>1 / k\}$. Let $\eta_{k}$ be a $C^{\infty}$-cutoff function satisfying supp $\eta_{k} \subset \Omega$ and

$$
0 \leq \eta_{k} \leq 1,\left.\quad \eta_{k}\right|_{\Omega_{k}}=1, \quad\left|\nabla \eta_{k}\right| \leq C k
$$

By the Sobolev embedding theorem, $u_{j} \rightarrow u$ in $L^{p}(\Omega)$; thus we can choose a subsequence $\left\{u_{j_{k}}\right\}$ such that, for all $k=1,2, \ldots$,

$$
\left(1+\left\|\nabla \eta_{k}\right\|_{\infty}\right)\left\|u_{j_{k}}-u\right\|_{L^{p}(\Omega)} \leq 1 / k
$$

Let $w_{k}=\eta_{k} u_{j_{k}}+\left(1-\eta_{k}\right) u$. Note that

$$
\nabla w_{k}= \begin{cases}\nabla u_{j_{k}} & \text { in } \Omega_{k}, \\ \eta_{k} \nabla u_{j_{k}}+\left(u_{j_{k}}-u\right) \otimes \nabla \eta_{k}+\left(1-\eta_{k}\right) \nabla u & \text { in } \Omega \backslash \Omega_{k}, \\ \nabla u & \text { in } D \backslash \Omega,\end{cases}
$$

where $a \otimes b$ stands for the rank-1 matrix $\left(a_{i} b_{j}\right)$. It is easy to see that $\left\{\left|\nabla w_{k}\right|\right\}$ is bounded in $L^{p}(D)$ and also equi-integrable in the case when $p=1$. Hence $w_{k} \rightarrow u$ in $W^{1, p}\left(D ; \mathbf{R}^{n}\right)$ and $\int_{\Omega}\left|\nabla u_{j_{k}}-\nabla w_{k}\right| \rightarrow 0$ as $k \rightarrow \infty$. Furthermore, by (1.1), we have $d_{\mathcal{K}}(\lambda X+Y) \leq K_{0} d_{\mathcal{K}}(X)+K_{0}+|Y|$ for $0 \leq \lambda \leq 1$ and thus

$$
\begin{aligned}
& I_{p}\left(w_{k} ; \Omega \backslash \Omega_{k}\right) \\
& \quad \leq C_{p}\left(I_{p}\left(u_{j_{k}} ; \Omega\right)+\left\|\nabla \eta_{k}\right\|_{\infty}^{p}\left\|u_{j_{k}}-u\right\|_{L^{p}(\Omega)}^{p}+\|\nabla u\|_{L^{p}\left(\Omega \backslash \Omega_{k}\right)}^{p}+\left|\Omega \backslash \Omega_{k}\right|\right),
\end{aligned}
$$

which, by (2.1), implies that $I_{p}\left(w_{k} ; D\right) \rightarrow 0$ as $k \rightarrow \infty$. Finally, the lemma follows by choosing a subsequence of $\left\{w_{k}\right\}$.

Corollary 2.2. Suppose $0 \in \mathcal{K}$ and $\Gamma_{1}=0$ in (1.4). Let $u_{j}$ be as given in the previous lemma, and let $p \in[\alpha, \beta]$. Then $u_{j} \rightarrow u$ strongly in $W_{\text {loc }}^{1, p}\left(\Omega ; \mathbf{R}^{n}\right)$ provided that $\nabla u_{j}(x) \rightarrow \nabla u(x)$ a.e. in $\Omega$.

Proof. Let $\Omega^{\prime} \subset \subset \Omega$ be given and let $k \gg 1$ so that $\Omega^{\prime} \subseteq \Omega_{k}$. Since $p \in[\alpha, \beta]$ and $w_{k}-u \in W_{0}^{1, p}\left(\Omega ; \mathbf{R}^{n}\right)$, by (1.5) we have

$$
\int_{\Omega^{\prime}}\left|\nabla u_{j_{k}}-\nabla u\right|^{p} \leq \int_{\Omega}\left|\nabla\left(w_{k}-u\right)\right|^{p} \leq \Gamma_{0}^{-1} \int_{\Omega} d_{\mathcal{K}}^{p}\left(\nabla w_{k}-\nabla u\right) .
$$


It is elementary to see that

$$
f_{k} \equiv 2^{p}\left(d_{\mathcal{K}}^{p}\left(\nabla w_{k}\right)+|\nabla u|^{p}\right)-d_{\mathcal{K}}^{p}\left(\nabla w_{k}-\nabla u\right)
$$

is nonnegative and tends to $2^{p}|\nabla u|^{p}$ a.e. in $\Omega$ as $k \rightarrow \infty$ if $\nabla u_{j} \rightarrow \nabla u$ a.e. in $\Omega$. Thus, by Fatou's lemma, one easily deduces that

$$
\limsup _{k \rightarrow \infty} \int_{\Omega} d_{\mathcal{K}}^{p}\left(\nabla w_{k}-\nabla u\right)=0
$$

which by (2.3) implies that $u_{j} \rightarrow u$ strongly in $W_{\text {loc }}^{1, p}\left(\Omega ; \mathbf{R}^{n}\right)$, since one can start with any subsequences of $\left\{u_{j}\right\}$.

\section{A Variational Principle and Higher Regularity}

Let $D$ be a domain in $\mathbf{R}^{m}$ and let $u$ be a map in $W^{1, p}\left(D ; \mathbf{R}^{n}\right)$. We define a complete metric space $(\mathcal{V}, \rho)$ by $\mathcal{V}=\mathcal{V}_{u, D}$ and $\rho=\rho_{D}$, where

$$
\mathcal{V}_{u, D} \equiv\left\{u+\zeta \mid \zeta \in W_{0}^{1,1}\left(D ; \mathbf{R}^{n}\right)\right\}, \quad \rho_{D}(w, v) \equiv \int_{D}|\nabla w-\nabla v| .
$$

Then, by Fatou's lemma, the functional $I_{p}(v ; D)$ is lower semicontinuous on $(\mathcal{V}, \rho)$ for all $p \geq 1$. The following variational principle and higher regularity result are crucial for proving our main theorem.

LEMma 3.1. For any $w_{k} \in \mathcal{V}$ with $I_{p}\left(w_{k} ; D\right) \leq \inf _{v \in \mathcal{V}} I_{p}(v ; D)+k^{-2} / 2$, there exists a $b_{k} \in \mathcal{V}$ such that $I_{p}\left(b_{k} ; D\right) \leq I_{p}\left(w_{k} ; D\right), \rho\left(b_{k}, w_{k}\right) \leq 1 / k$, and

$$
I_{p}\left(b_{k} ; D\right)<I_{p}(w ; D)+k^{-1} \rho\left(w, b_{k}\right) \quad \forall w \in \mathcal{V}, \quad w \neq b_{k} .
$$

Proof. This lemma is a special version of a general Ekeland's variational principle [3]; see [2, Thm. 4.2].

Note that it is a direct consequence of the coercivity condition (1.5) that if $p \in$ $[\alpha, \beta]$ then sequences $\left\{w_{k}\right\}$ and $\left\{b_{k}\right\}$ in Lemma 3.1 are both uniformly bounded in $W^{1, p}\left(D ; \mathbf{R}^{n}\right)$. Furthermore, we have the following theorem.

THEOREM 3.2. There exist $\varepsilon_{n}>0$ and integer $N_{n}$ depending only on $n, \alpha, \beta$, and $\mathcal{K}$ such that, if $p \in[\alpha, \beta]$, the sequence $\left\{b_{k}\right\}$ determined in the previous lemma must then satisfy

$$
\sup _{k \geq N_{n}} \int_{D^{\prime}}\left|\nabla b_{k}\right|^{p+\varepsilon_{n}}<\infty \quad \forall D^{\prime} \subset \subset D .
$$

Proof. We first prove that the reverse Hölder inequalities

$$
\begin{aligned}
& f_{B_{R}}\left|\nabla b_{k}\right|^{p} \\
& \quad \leq \beta_{n}\left(f_{B_{2 R}}\left|\nabla b_{k}\right|^{p n /(n+p)}\right)^{(n+p) / n}+\gamma_{n} \quad \forall B_{2 R}=B(a, 2 R) \subset \subset D
\end{aligned}
$$


hold for all $k \geq N_{n}$, where $N_{n}, \beta_{n}$, and $\gamma_{n}$ are constants depending on $n, \alpha, \beta$, and $\mathcal{K}$. We follow the idea used in [16]. In the following, we use $c_{0}, c_{1}, \ldots$ to denote the constants depending only on $n, \alpha, \beta$, and $\mathcal{K}$. Let $B_{2 R}=B(a, 2 R) \subset \subset$ $D$ and $0<s<t \leq 2 R$. Let $\eta \in C_{0}^{\infty}(D)$ be a cutoff function such that

$$
0 \leq \eta \leq 1,\left.\quad \eta\right|_{B_{s}}=1,\left.\quad \eta\right|_{D \backslash B_{t}}=0, \quad|\nabla \eta| \leq c_{0}(t-s)^{-1} .
$$

Let $w=\eta v+(1-\eta) b_{k}$ and $\phi=b_{k}-w$, where $v$ is a constant to be chosen later. Then $w \in \mathcal{V}=\mathcal{V}_{u, D}, \phi \in W_{0}^{1, p}\left(B_{t} ; \mathbf{R}^{n}\right)$, and

$$
\nabla w=(1-\eta) \nabla b_{k}-\left(b_{k}-v\right) \otimes \nabla \eta, \quad \nabla \phi=\eta \nabla b_{k}+\left(b_{k}-v\right) \otimes \nabla \eta .
$$

Using the inequality $d_{\mathcal{K}}^{p}(X+Y) \leq 2^{\beta}\left(d_{\mathcal{K}}^{p}(X)+|Y|^{p}\right)$, by (1.1) and (1.4) we have

$$
\begin{aligned}
\int_{B_{s}}\left|\nabla b_{k}\right|^{p} & \leq \int_{B_{t}}|\nabla \phi|^{p} \leq \Gamma_{0}^{-1} \int_{B_{t}} d_{\mathcal{K}}^{p}(\nabla \phi)+\Gamma_{1}\left|B_{t}\right| \\
& \leq c_{1} \int_{B_{t}} d_{\mathcal{K}}^{p}\left(\nabla b_{k}\right)+\frac{c_{1}}{(t-s)^{p}} \int_{B_{t} \backslash B_{s}}\left|b_{k}-v\right|^{p}+c_{1}\left|B_{t}\right| .
\end{aligned}
$$

Since $\nabla w=\nabla b_{k}$ in $D \backslash B_{t}$ and $\nabla w=0$ in $B_{s}$, the first term in (3.6) can be estimated by (3.2) as

$$
\int_{B_{t}} d_{\mathcal{K}}^{p}\left(\nabla b_{k}\right) \leq \int_{B_{t} \backslash B_{s}} d_{\mathcal{K}}^{p}(\nabla w)+d_{\mathcal{K}}^{p}(0)\left|B_{s}\right|+k^{-1} \int_{B_{t}}\left|\nabla w-\nabla b_{k}\right|
$$

We now use (3.5) and the inequality $d_{\mathcal{K}}(X) \leq|X|+d_{\mathcal{K}}(0)$ to deduce

$$
\begin{aligned}
& \int_{B_{t} \backslash B_{s}} d_{\mathcal{K}}^{p}(\nabla w) \\
& \leq c_{2} \int_{B_{t} \backslash B_{s}}\left|\nabla b_{k}\right|^{p}+\frac{c_{2}}{(t-s)^{p}} \int_{B_{t} \backslash B_{s}}\left|b_{k}-v\right|^{p}+c_{2}\left|B_{t} \backslash B_{s}\right| .
\end{aligned}
$$

Combining (3.6)-(3.8), we have

$$
\begin{aligned}
\int_{B_{s}}\left|\nabla b_{k}\right|^{p} & \leq c_{3} \int_{B_{t} \backslash B_{s}}\left|\nabla b_{k}\right|^{p}+\frac{c_{3}}{(t-s)^{p}} \int_{B_{2 R}}\left|b_{k}-\nu\right|^{p} \\
& +\frac{c_{3}}{k} \int_{B_{t}}\left|\nabla b_{k}-\nabla w\right|+c_{3}\left|B_{2 R}\right| .
\end{aligned}
$$

Note also that, since $t \leq t^{p}+1$ for all $t \geq 0$ and $p \geq 1$,

$$
\begin{aligned}
\int_{B_{t}}\left|\nabla b_{k}-\nabla w\right|= & \int_{B_{s}}\left|\nabla b_{k}\right|+\int_{B_{t} \backslash B_{s}}|\nabla \phi| \\
\leq & \int_{B_{s}}\left|\nabla b_{k}\right|^{p}+c_{4} \int_{B_{t} \backslash B_{s}}\left|\nabla b_{k}\right|^{p} \\
& +\frac{c_{4}}{(t-s)^{p}} \int_{B_{2 R}}\left|b_{k}-\nu\right|^{p}+c_{4}\left|B_{2 R}\right| .
\end{aligned}
$$

We now choose $N_{n}=2 c_{3}$. Then, for $k \geq N_{n}$, by (3.9) and (3.10) we have

$$
\int_{B_{s}}\left|\nabla b_{k}\right|^{p} \leq c_{5} \int_{B_{t} \backslash B_{s}}\left|\nabla b_{k}\right|^{p}+\frac{c_{5}}{(t-s)^{p}} \int_{B_{2 R}}\left|b_{k}-v\right|^{p}+c_{5}\left|B_{2 R}\right| .
$$


Filling the hole - that is, adding $c_{5} \int_{B_{s}}\left|\nabla b_{k}\right|^{p}$ to both sides of (3.11)-we obtain

$$
\int_{B_{s}}\left|\nabla b_{k}\right|^{p} \leq \frac{c_{5}}{1+c_{5}} \int_{B_{t}}\left|\nabla b_{k}\right|^{p}+\frac{c_{6}}{(t-s)^{p}} \int_{B_{2 R}}\left|b_{k}-v\right|^{p}+c_{6}\left|B_{2 R}\right| .
$$

This inequality holds for all $0<s<t \leq 2 R$. Thus, a standard iteration argument [6] yields

$$
\int_{B_{R}}\left|\nabla b_{k}\right|^{p} \leq c_{7} R^{-p} \int_{B_{2 R}}\left|b_{k}-v\right|^{p}+c_{7}\left|B_{2 R}\right|
$$

and hence

$$
f_{B_{R}}\left|\nabla b_{k}\right|^{p} \leq \frac{c_{8}}{R^{n+p}} \int_{B_{2 R}}\left|b_{k}-v\right|^{p}+c_{8}
$$

Now choose

$$
v=v_{R}=f_{B_{2 R}} b_{k}
$$

and use the Sobolev-Poincaré inequality

$$
\int_{B_{2 R}}\left|b_{k}-v_{R}\right|^{p} \leq \sigma_{n}\left(\int_{B_{2 R}}\left|\nabla b_{k}\right|^{p n /(n+p)}\right)^{(n+p) / n}
$$

in (3.13), and we obtain (3.4).

To continue the proof, we let $f_{k}=1+\left|\nabla b_{k}\right|^{p n /(n+p)}$ and $r=(n+p) / n$. Then, by (1.4), $\left\{f_{k}\right\}$ is bounded in $L^{r}(D)$ and, for all $k \geq N_{n}$, by (3.4) we have

$$
f_{B_{R}} f_{k}^{r} \leq \kappa_{n}\left(f_{B_{2 R}} f_{k}\right)^{r} \quad \forall B_{2 R} \subset \subset D
$$

where $\kappa_{n}$ is a constant depending on $n, \alpha, \beta$, and $\mathcal{K}$. Therefore, by [8, Prop. 6.1] and [6, Thm. 6.1], $\left\{f_{k}\right\}$ is bounded in $L_{\mathrm{loc}}^{s}(D)$ for $s=r+(r-1) / 10^{n+r} 4^{n} \kappa_{n}^{r}$ and hence $\left\{b_{k}\right\}$ is bounded in $W_{\text {loc }}^{1, p+\varepsilon}(D)$ with

$$
\varepsilon=\varepsilon(p)=\frac{p^{2}}{10^{n+1} 4^{n} \kappa_{n}(n+p)\left(10 \kappa_{n}\right)^{p / n}} .
$$

Let $\varepsilon_{n}=\min \{\varepsilon(p) \mid \alpha \leq p \leq \beta\}$. Then it is easily seen that $\varepsilon_{n}>0$, and (3.3) follows.

\section{Proof of Theorem 1.1}

Let $u_{0} \in W_{\text {loc }}^{1, \alpha}\left(D_{0} ; \mathbf{R}^{n}\right)$ be the map given before, satisfying (1.3). Let $\left\{u_{j}\right\}$ be any sequence in $W^{1, \alpha}\left(\Omega ; \mathbf{R}^{n}\right)$ that satisfies

$$
u_{j} \rightarrow u_{0} \text { weakly in } W^{1, \alpha}\left(\Omega ; \mathbf{R}^{n}\right) \text { and } \lim _{j \rightarrow \infty} I_{\alpha}\left(u_{j} ; \Omega\right)=0 .
$$

In the following, let $\left\{D_{i}\right\}(i=1,2, \ldots)$ be an arbitrary sequence of subdomains of $D_{0}$ that satisfies

$$
\Omega \subset \subset D_{i+1} \subset \subset D_{i} \subset \subset D_{0}, \quad i=1,2, \ldots .
$$

We proceed in several steps. 
Step 1. Let $\left\{w_{k}\right\}$ be the sequence determined in Lemma 2.1 from $\left\{u_{j}\right\}$ with $p=\alpha, u=u_{0}$, and $D=D_{1}$. Using $D_{1}$ and $u_{0} \in W^{1, \alpha}\left(D ; \mathbf{R}^{n}\right)$, we define space $(\mathcal{V}, \rho)=\left(\mathcal{V}_{u_{0}, D_{1}}, \rho_{D_{1}}\right)$ as in Section 3. Then $w_{k} \in \mathcal{V}$ and, since $I_{\alpha}\left(w_{k} ; D_{1}\right) \leq$ $k^{-2} / 2$, it is clear that inf $I_{\mathcal{V}}\left(v ; D_{1}\right)=0$; hence we can apply Lemma 3.1 to the sequence $\left\{w_{k}\right\}$. Let $\left\{b_{k}\right\}$ be the corresponding sequence in $\mathcal{V}$ satisfying $\rho_{D_{1}}\left(w_{k}, b_{k}\right) \leq$ $1 / k$. In the following steps, we study properties of this new sequence $\left\{b_{k}\right\}$.

Step 2. By Theorem 3.2, $\left\{b_{k}\right\}\left(k \geq N_{n}\right)$ is bounded in $W_{\text {loc }}^{1, \alpha+\varepsilon_{n}}\left(D_{1} ; \mathbf{R}^{n}\right)$, where $N_{n}$ and $\varepsilon_{n}$ are the absolute constants determined in Theorem 3.2. Therefore, $\left\{b_{k}\right\}$ converges weakly in $W^{1, \alpha+\varepsilon_{n}}\left(\Omega ; \mathbf{R}^{n}\right)$. Since $\rho_{D_{1}}\left(w_{k}, b_{k}\right) \leq 1 / k$ and $w_{k} \rightarrow$ $u_{0}$ in $W^{1, \alpha}\left(D_{1} ; \mathbf{R}^{n}\right)$, we deduce that $b_{k} \rightarrow u_{0}$ in $W_{\text {loc }}^{1, \alpha+\varepsilon_{n}}\left(D_{1} ; \mathbf{R}^{n}\right)$. This readily implies $u_{0} \in W^{1, \alpha+\varepsilon_{n}}\left(D_{2} ; \mathbf{R}^{n}\right)$. In what follows, let $\varepsilon_{0}=\varepsilon_{n} / 2$. We claim $\lim _{k \rightarrow \infty} I_{\alpha+\varepsilon_{0}}\left(b_{k} ; \Omega\right)=0$. To see this, we use the elementary estimate

$$
d_{\mathcal{K}}^{\alpha+\varepsilon_{0}}(X) \leq \delta\left(|X|^{\alpha+2 \varepsilon_{0}}+1\right)+C_{\delta} d_{\mathcal{K}}^{\alpha}(X) \quad \forall \delta>0
$$

with $X=\nabla b_{k}$, and integrate it over $\Omega$ to obtain

$$
I_{\alpha+\varepsilon_{0}}\left(b_{k} ; \Omega\right) \leq \delta \int_{\Omega}\left(\left|\nabla b_{k}\right|^{\alpha+2 \varepsilon_{0}}+1\right)+C_{\delta} I_{\alpha}\left(b_{k} ; \Omega\right) .
$$

Note that, from Theorem 3.2,

$$
\sup _{k \geq N_{n}} \int_{\Omega}\left|\nabla b_{k}\right|^{\alpha+2 \varepsilon_{0}} \leq M<\infty, \quad \lim _{k \rightarrow \infty} I_{\alpha}\left(b_{k} ; D_{1}\right)=0 .
$$

Let $k \rightarrow \infty$ in (4.2). We then have $\lim \sup _{k \rightarrow \infty} I_{\alpha+\varepsilon_{0}}\left(b_{k} ; \Omega\right) \leq \delta M$ for all $\delta>0$, which implies that

$$
\lim _{k \rightarrow \infty} I_{\alpha+\varepsilon_{0}}\left(b_{k} ; \Omega\right)=0 .
$$

Also, by Lemmas 2.1 and 3.1, we have $\lim _{k \rightarrow \infty} \int_{\Omega}\left|\nabla u_{j_{k}}-\nabla b_{k}\right|=0$.

Step 3. In Step 2, we proved that $u_{0} \in W^{1, \alpha+\varepsilon_{0}}\left(D_{2} ; \mathbf{R}^{n}\right)$ and obtained a new sequence $\left\{b_{k}^{(1)}\right\}=\left\{b_{k}\right\}$ in $W^{1, \alpha+\varepsilon_{0}}\left(\Omega ; \mathbf{R}^{n}\right)$. This sequence satisfies

$$
\lim _{k \rightarrow \infty} \int_{\Omega}\left|\nabla u_{j_{k}}-\nabla b_{k}^{(1)}\right|=0
$$

for a subsequence $\left\{u_{j_{k}}\right\}$ of the original sequence $\left\{u_{j}\right\}$ and also satisfies

$$
b_{k}^{(1)} \rightarrow u_{0} \text { weakly in } W^{1, \alpha+\varepsilon_{0}}\left(\Omega ; \mathbf{R}^{n}\right) \text { and } \lim _{k \rightarrow \infty} I_{\alpha+\varepsilon_{0}}\left(b_{k}^{(1)} ; \Omega\right)=0 .
$$

Hence $\left\{b_{k}^{(1)}\right\}$ satisfies the same type of conditions (4.1) as satisfied by $\left\{u_{j}\right\}$ except that now it is in a better space, $W^{1, \alpha+\varepsilon_{0}}\left(\Omega ; \mathbf{R}^{n}\right)$. Therefore, if $\alpha+\varepsilon_{0} \leq \beta$, we can apply Step 1 again with $\left\{b_{k}^{(1)}\right\}$ replacing $\left\{u_{j}\right\}, D_{2}$ replacing $D_{1}$, and $\alpha+\varepsilon_{0}$ replacing $\alpha$. Now let integer $N$ and number $\bar{\varepsilon}>0$ be determined by

$$
\alpha+(N-1) \varepsilon_{0} \leq \beta<\alpha+N \varepsilon_{0}, \quad \alpha+N \varepsilon_{0}=\beta+\bar{\varepsilon} .
$$

We repeat this step $N$ times to eventually prove $u_{0} \in W^{1, \beta+\bar{\varepsilon}}\left(D_{N+1} ; \mathbf{R}^{n}\right)$ and obtain a sequence $\left\{b_{v}^{(N)}\right\}$ in $W^{1, \beta+\bar{\varepsilon}}\left(\Omega ; \mathbf{R}^{n}\right)$ that satisfies both 


$$
b_{\nu}^{(N)} \rightarrow u_{0} \text { weakly in } W^{1, \beta+\bar{\varepsilon}}\left(\Omega ; \mathbf{R}^{n}\right) \text { and } \lim _{\nu \rightarrow \infty} I_{\beta+\bar{\varepsilon}}\left(b_{\nu}^{(N)} ; \Omega\right)=0
$$

and also $\lim _{\nu \rightarrow \infty} \int_{\Omega}\left|\nabla u_{j}^{(v)}-\nabla b_{v}^{(N)}\right|=0$, where $\left\{u_{j}^{(v)}\right\}$ is a subsequence of the original sequence $\left\{u_{j}\right\}$.

Step 4. Finally, let $\left\{v_{k}\right\}$ be the sequence in $W^{1, \beta+\bar{\varepsilon}}\left(D_{N+1} ; \mathbf{R}^{n}\right)$ determined from $\left\{b_{v}^{(N)}\right\}$ in the same way that $\left\{w_{k}\right\}$ was from $\left\{u_{j}\right\}$ in Lemma 2.1. We extend $\left\{v_{k}\right\}$ to $D_{0} \backslash D_{N+1}$ by $u_{0}$. Then, this sequence satisfies all the requirements of Theorem 1.1 and thus proves the theorem.

\section{Strong Convergence of Weakly Almost Conformal Mappings}

We now consider the so-called conformal set

$$
\mathcal{K}=C_{n}=\{\lambda R \mid \lambda \geq 0, R \in \mathrm{SO}(n)\},
$$

where $\mathrm{SO}(n)$ is the set of all orthogonal matrices of determinant 1 . Since $\mathcal{K}=C_{n}$ is a closed cone, condition (1.1) is satisfied.

Recall that a map $\varphi$ on the extended space $\mathbf{R}^{n} \cup\{\infty\}$ is a Möbius transformation if it is a composition of finitely many similarities and inversions with respect to the sphere $[12 ; 13]$. A sequence $\left\{u_{j}\right\}$ in $W^{1, p}\left(\Omega ; \mathbf{R}^{n}\right)$ is said to be (weakly if $p<n$ ) almost conformal if

$$
\lim _{j \rightarrow \infty} \int_{\Omega} d_{C_{n}}^{p}\left(\nabla u_{j}(x)\right) d x=0 .
$$

As an application of Theorem 1.1, we prove in this section the following strong convergence result concerning the weakly almost conformal sequences. See also $[11 ; 14 ; 16]$.

THEOREM 5.1. There exists a number $p<n$ such that any sequence $\left\{u_{j}\right\}$ converging weakly in $W^{1, p}\left(\Omega ; \mathbf{R}^{n}\right)$ and satisfying (5.2) must converge strongly to a Möbius transformation in both $W^{1,1}\left(\Omega ; \mathbf{R}^{n}\right)$ and $W_{\mathrm{loc}}^{1, p}\left(\Omega ; \mathbf{R}^{n}\right)$.

Remarks. (1) It follows from Yan [14] that any number $p$ that validates Theorem 5.1 must be at least the half-dimension, that is, $p \geq n / 2$. Note also that the strong convergence in $W^{1, p}\left(\Omega ; \mathbf{R}^{n}\right)$ for $p \geq n$ follows easily from a theorem of Evans and Gariepy [4, Thm. 1]. See also the proof of Proposition 5.5.

(2) If the dimension $n=2 l$ is even, Müller, Šverák, and Yan [11] proved that the smallest such $p$ is precisely the half-dimension $l=n / 2$. A key ingredient, as observed by Iwaniec and Martin [9], is that in this case the conformality of a map can be characterized by a nonlinear Cauchy-Riemann equation involving the determinants of $l \times l$ sub-Jacobians, which enables us to use standard elliptic estimates and the compensated compactness method; see [11] for details.

(3) Because the equations (the so-called Beltrami systems) governing the conformal mappings in the case of odd dimensions are essentially nonlinear, the methods used in [11] do not apply and in this case the problem regarding the smallest $p$ validating Theorem 5.1 remains unsolved; see also $[7 ; 9 ; 16]$. 
In order to prove Theorem 5.1, we will need the following lemmas.

Lemma 5.2. There exist $p_{0}<n$ and $\Gamma>0$ such that, for every ball $B \subset \mathbf{R}^{n}$,

$$
\int_{B} d_{C_{n}}^{p}(\nabla \phi) \geq \Gamma \int_{B}|\nabla \phi|^{p} \quad \forall p \in\left[p_{0}, n\right], \phi \in C_{0}^{\infty}\left(B ; \mathbf{R}^{n}\right) .
$$

Proof. This has been proved in Yan [14, Thm. 1.3] using the estimates of very weak solutions of $p$-harmonic equations established in Iwaniec [7]; see also [10] and [16, Cor. 3.3]. We only remark here that $p_{0} \geq n / 2$, from [14, Thm. 1.4].

LEMMA 5.3. There is a $p_{1}<n$ such that, for any sequence $\left\{u_{j}\right\}$ as given in Theorem 5.1 with $p \in\left[p_{1}, n\right]$, the weak limit $\bar{u}$ must be a restriction of an orientationpreserving Möbius transformation onto $\Omega$.

Proof. By [16, Cor. C], we know $p_{1}<n$ can be chosen such that any weak limit $\bar{u}$ as given in the lemma must be a weakly conformal map; that is, $\vec{u}$ satisfies

$$
\nabla \bar{u}(x) \in C_{n} \text { a.e. } x \in \Omega \text {. }
$$

We can choose $p_{1}<n$ even closer to $n$ so that the generalized Liouville's theorem in Iwaniec [7, Thm. 3] will assert that $\bar{u}$ must be a restriction of an orientationpreserving Möbius transformation onto $\Omega$. The proof is thus complete.

LEMMA 5.4. If $p \geq n / 2$ and $\partial \Omega$ is sufficiently smooth, then any Möbius transformation that belongs to $W^{1, p}\left(\Omega ; \mathbf{R}^{n}\right)$ must be a $C^{\infty}$-diffeomorphism of a neighborhood $D$ of $\bar{\Omega}$ into $\mathbf{R}^{n}$.

Proof. Let $\delta$ and $\mu$ be defined by

$$
\delta(x) \equiv A x+b, \quad \mu(x) \equiv a+r^{2}|x-a|^{-2}(x-a) .
$$

A similarity is a transformation $\delta$ with $A=\lambda P$ for some $\lambda \in \mathbf{R}$ and orthogonal matrix $P$. By a representation result in [13, p. 75], a Möbius transformation $\varphi$ is either a similarity or a transformation representable as $\varphi=\delta \circ \mu$, with $\delta, \mu$ defined by (5.3) and $A$ orthogonal.

Let $\varphi$ be a Möbius transformation and let $\varphi \in W^{1, p}\left(\Omega ; \mathbf{R}^{n}\right)$. If $\varphi$ is a similarity then it extends to the whole $\mathbf{R}^{n}$. Suppose now that $\varphi$ is given by $\varphi=\delta \circ \mu$, where $\delta$ and $\mu$ are defined by (5.3) and $A$ is orthogonal. Then

$$
\nabla \varphi(x)=r^{2}|x-a|^{-2} A\left(I-2 \frac{x-a}{|x-a|} \otimes \frac{x-a}{|x-a|}\right) .
$$

Thus

$$
|\nabla \varphi(x)|=\sqrt{n} r^{2}|x-a|^{-2} .
$$

Suppose $\partial \Omega$ is sufficiently smooth; then $\varphi \in W^{1, p}\left(\Omega ; \mathbf{R}^{n}\right)$ only for $1 \leq p<n / 2$ if $a \in \bar{\Omega}$. Thus, if $p \geq n / 2$ and $\varphi \in W^{1, p}\left(\Omega ; \mathbf{R}^{n}\right)$, we have $a \notin \bar{\Omega}$ and hence $\varphi \in$ $C^{\infty}\left(D ; \mathbf{R}^{n}\right)$ for some domain $D$ containing $\bar{\Omega}$. The lemma is proved.

In the following, we let $p_{*}=\max \left\{p_{0}, p_{1}\right\}$. Then $n / 2 \leq p_{*}<n$. Let $p \in\left[p_{*}, n\right]$, let $\left\{u_{j}\right\}$ be a sequence in $W^{1, p}\left(\Omega ; \mathbf{R}^{n}\right)$ as given in the theorem, and let $\bar{u}$ be the weak 
limit. It follows from Lemmas 5.2-5.4 that $\bar{u}$ extends to a $C^{\infty}$-diffeomorphism in a neighborhood $D$ of $\bar{\Omega}$ as an orientation-preserving Möbius transformation. Thus $\nabla \bar{u}(x) \in C_{n}$ for all $x \in D$. Consequently, all conditions of Theorem 1.1 are satisfied with $\alpha=p_{*}, \beta=n, D_{0}=D$, and $u_{0}=\bar{u}$. Therefore, by Theorem 1.1, we obtain a sequence $\left\{v_{k}\right\}$ in $W_{\text {loc }}^{1, n+\bar{\varepsilon}}\left(D ; \mathbf{R}^{n}\right)$ that satisfies $v_{k}=\bar{u}$ in $D \backslash \Omega$ and $\int_{\Omega}\left|\nabla v_{k}-\nabla u_{j_{k}}\right| \rightarrow 0$ as $k \rightarrow \infty$ for some subsequence $\left\{u_{j_{k}}\right\}$; moreover,

$$
v_{k} \rightarrow \bar{u} \text { in } W_{\mathrm{loc}}^{1, n+\bar{\varepsilon}}\left(D ; \mathbf{R}^{n}\right) \text { and } \lim _{k \rightarrow \infty} \int_{D} d_{C_{n}}^{n+\bar{\varepsilon}}\left(\nabla v_{k}\right)=0 .
$$

Proposition 5.5. We have $v_{k} \rightarrow \bar{u}$ strongly in $W^{1, n}\left(\Omega ; \mathbf{R}^{n}\right)$.

Proof. Since det $\nabla u$ is a null Lagrangian [1], by (5.4) we have

$$
\lim _{k \rightarrow \infty} \int_{\Omega} \operatorname{det} \nabla v_{k}=\int_{\Omega} \operatorname{det} \nabla \bar{u} .
$$

Consider the function

$$
G(X)=|X|^{n}-n^{n / 2} \operatorname{det} X .
$$

Note that, by Hadamard's inequality, $G(X) \geq 0$ and $G(X)=0$ if and only if $X \in$ $C_{n}$. By homogeneity, $G(X) \leq \tau|X|^{n}+C_{\tau} d_{C_{n}}^{n}(X)$ for all $\tau>0$. Thus, by (5.4), we have

$$
\lim _{k \rightarrow \infty} \int_{D} G\left(\nabla v_{k}\right)=0=\int_{D} G(\nabla \bar{u})
$$

combined with (5.5), this yields $\int_{\Omega}\left|\nabla v_{k}\right|^{n} \rightarrow \int_{\Omega}|\nabla \bar{u}|^{n}$ and hence $\nabla v_{k} \rightarrow \nabla \bar{u}$ strongly in $L^{n}\left(\Omega ; \mathcal{M}^{n \times n}\right)$. The proof is complete.

Note that the strong convergence as asserted in the proposition also follows from (5.4) and (5.7) by the result [4, Thm. 1], since $G(X)$ is uniformly strictly $W^{1, n_{-}}$ quasiconvex in the sense defined by [4].

Proof of Theorem 5.1. By Proposition 5.5, the subsequence $\left\{u_{j_{k}}\right\}$ determined as above converges strongly to $\bar{u}$ in $W^{1,1}\left(\Omega ; \mathbf{R}^{n}\right)$, so $\nabla u_{j_{k}}(x) \rightarrow \nabla \bar{u}(x)$ for a.e. $x \in$ $\Omega$. Therefore, by Corollary 2.2 and Lemma 5.2, $u_{j_{k}} \rightarrow \bar{u}$ strongly in $W_{\text {loc }}^{1, p}\left(\Omega ; \mathbf{R}^{n}\right)$. Since we can start with arbitrary subsequences of $\left\{u_{j}\right\}$, we see that the original sequence $\left\{u_{j}\right\}$ converges strongly to $\bar{u}$ in both $W^{1,1}\left(\Omega ; \mathbf{R}^{n}\right)$ and $W_{\text {loc }}^{1, p}\left(\Omega ; \mathbf{R}^{n}\right)$. The proof of Theorem 5.1 is now complete.

\section{References}

[1] J. M. Ball, Convexity conditions and existence theorems in nonlinear elasticity, Arch. Rational Mech. Anal. 63 (1977), 337-403.

[2] D. G. De Figueiredo, Lectures on the Ekeland variational principle with applications and detours, Tata Institute lecture. Springer, New York, 1989.

[3] I. Ekeland, On the variational principle, J. Math. Anal. Appl. 47 (1974), 324-353.

[4] L. C. Evans and R. F. Gariepy, Some remarks concerning quasiconvexity and strong convergence, Proc. Roy. Soc. Edinburgh Sect. A 106 (1987), 53-61. 
[5] F. W. Gehring, The $L^{p}$-integrability of the partial derivatives of a quasiconformal mapping, Acta Math. 130 (1973), 265-277.

[6] M. Giaquinta, Multiple integrals in the calculus of variations and nonlinear elliptic systems, Princeton Univ. Press, Princeton, NJ, 1983.

[7] T. Iwaniec, p-Harmonic tensors and quasiregular mappings, Ann. of Math. (2) 136 (1992), 589-624.

[8] - The Gehring lemma, preprint.

[9] T. Iwaniec and G. Martin, Quasiregular mappings in even dimensions, Acta Math. 170 (1993), 29-81.

[10] T. Iwaniec and C. Sbordone, Weak minima of variational integrals, J. Reine Angew. Math. 454 (1994), 143-161.

[11] S. Müller, V. Šverák, and B. Yan, Sharp stability results for almost conformal maps in even dimensions, J. Geom. Anal. (to appear).

[12] Y. G. Reshetnyak, Space mappings with bounded distortion, Transl. Math. Monographs, 73, Amer. Math. Soc., Providence, RI, 1989.

[13] - Stability theorems in geometry and analysis, Kluwer, Dordrecht, 1994.

[14] B. Yan, Remarks about $W^{1, p}$-stability of the conformal set in higher dimensions, Ann. Inst. H. Poincaré Anal. Non Linéaire 13 (1996), 691-705.

[15] - On rank-one convex and polyconvex conformal energy functions with slow growth, Proc. Roy. Soc. Edinburgh Sect. A 127 (1997), 651-663.

[16] B. Yan and Z. Zhou, Stability of weakly almost conformal mappings, Proc. Amer. Math. Soc. (to appear).

B. Yan

Department of Mathematics

Michigan State University

East Lansing, MI 48824

yan@math.msu.edu
Z. Zhou

Department of Mathematics

Michigan State University

East Lansing, MI 48824

zfzhou@math.msu.edu 
